\title{
SECAGEM POR ATOMIZAÇÃO DA POLPA DE YACON (SMALLANTHUS SONCHIFOLIUS)
}

\author{
J. C. C. SANTOS ${ }^{1}$, S. P. OLIVEIRA ${ }^{1}$, C. A. SANTOS ${ }^{1}$, T.P.V.B. \\ Dias $^{1}$, L. F. OLIVEIRA ${ }^{1}$ \\ ${ }^{1}$ Universidade Federal de São João del Rei \\ E-mail para contato: jhenifercristinacs@gmail.com
}

\begin{abstract}
RESUMO - O yacon (Smallanthus sonchifolius) vem despertando grande interesse no meio científico devido as suas propriedades funcionais, sendo considerado benéfico à saúde, pela ação antioxidante, melhora na absorção de minerais, ação como prebiótico e, principalmente, pelo efeito hipoglicêmico. Mas, além de sazonal, possui elevada atividade de água, o que resulta em uma curta vida de prateleira. $\mathrm{O}$ processo de secagem por atomização é uma alternativa para a preservação de alimentos com alto valor agregado. Este trabalho avaliou a influência da temperatura e vazão na microencapsulação por Spray dryer da polpa do yacon. Os encapsulados foram caracterizados quanto à higroscopicidade, solubilidade e teor de compostos fenólicos. Os ensaios apresentaram ótimos resultados de solubilidade $(85,063 \%$ a $100,00 \%$ ), obtendo um rendimento de secagem de até 55,103\%. Observou-se que a vazão foi significativa em relação aos compostos fenólicos e higroscopicidade.
\end{abstract}

\section{INTRODUÇÃO}

O yacon é um tubérculo que se destaca pelas suas características funcionais como ação prebiótica, por possuir como carboidrato de reserva frutooligosacarideos (FOS). A sua ingestão pode trazer benefícios como, efeito hipoglicêmico, ação anti-inflamatória, antioxidante e atua na redução do risco carcinogênico (Takenaka et al. 2003; Delgado et al. 2013).

No entanto, sua colheita é sazonal, é um produto altamente perecível, por apresentar uma elevada atividade de água, e apresenta um significativo decréscimo em seu conteúdo de FOS durante o armazenamento e no transporte (Castro et al., 2012; Scheid et al., 2013).

Técnicas de secagem por atomização podem ser uma excelente alternativa para secagem de alimentos com alto valor agregado, como o yacon, pois tem a capacidade de transformar os extratos aquosos em pó, gerando produtos com maior estabilidade e preservando seus compostos biologicamente ativos.

Assim, este trabalho, objetivou avaliar o efeito da temperatura e vazão de alimentação em Spray dryer, da polpa de yacon. Afim de, buscar um produto seco que gere um maior rendimento de secagem, associado com a maior durabilidade e conservação dos compostos bioativos em relação ao produto fresco. 


\section{METODOLOGIA EXPERIMENTAL}

\section{1. Preparo das matérias primas}

Após higienizados e sanitizados os tubérculos de yacon, adiquiridos no mercado local de Divinópolis, MG, foram descascados manualmente para utilização da polpa no preparo do extrato para secagem em Spray Dryer. O extrato da polpa foi realizado conforme Nish (2012). Fatias de yacon foram trituradas com $0,03 \%$ de bissulfito de sódio, com relação aos sólidos totais, durante 2 minutos. Por fim, filtrou-se o material com filtro de organza.

\subsection{Secagem em Spray dryer}

Para secagem o adjuvante, goma arábica, na concentração de $34 \%$ (sendo a sua concentração definida em função da concentração de sólidos totais do extrato) foi incorporado ao extrato e deixado sob agitação por 30 minutos. Após a obtenção dos extratos, foram realizadas as secagens em Mini Spray dryer modelo MSDi 1.0 Labmaq, com bico atomizador de 1,0 mm de diâmetro, pressão de ar comprimido de $3,5 \mathrm{kgf} / \mathrm{cm}^{2}$ e vazão do ar de atomização de 40,5 L/min.

Para cada experimento variou-se a vazão de alimentação e a temperatura do ar de secagem, definindo em planejamento $2^{2}$ com três pontos centrais, conforme Tabela 1.

Tabela 1 - Delineamento experimental para a secagem do extrato do yacon

\begin{tabular}{ccccc}
\hline Ensaios & \multicolumn{2}{c}{ Variáveis codificadas } & \multicolumn{2}{c}{ Variáveis reais } \\
\hline 1 & $\mathrm{X} 1$ & $\mathrm{X} 2$ & $\mathrm{~T}\left[{ }^{\circ} \mathrm{C}\right]$ & $\mathrm{W}[\mathrm{L} / \mathrm{h}]$ \\
\hline 2 & -1 & -1 & 120 & 0,38 \\
3 & 1 & -1 & 158 & 0,38 \\
4 & -1 & 1 & 120 & 0,82 \\
5 & 1 & 1 & 158 & 0,82 \\
6 & 0 & 0 & 139 & 0,60 \\
7 & 0 & 0 & 139 & 0,60 \\
& 0 & 0 & 139 & 0,60 \\
\hline
\end{tabular}

Onde T é a temperatura, W, a vazão de alimentação do secador.

Após secagem, os pós atomizados foram caracterizados com relação à solubilidade (CanoChauca et al., 2005), higroscopicidade (Tonon et al., 2009), e teor de compostos fenólicos (Singleton et al., 1999), todas as análises foram realizadas em triplicata.

O rendimento dos processos de secagem foi calculado conforme Medeiros (2001). A análise estatística dos dados foi realizada com auxílio do software Statistica 8.0, (STATISTICA, 2008). Considerou-se um nível de confiabilidade igual a $95 \%$ e realizou-se o teste $\mathrm{F}$ para a análise do ajuste da regressão.

\section{RESULTADOS E DISCUSSÕES}


Os resultados obtidos do processo de secagem do extrato de yacon estão representados na Tabela 2, onde observa-se que o pó de yacon apresentou grande solubilidade, o que o faz um potencial componente em formulações de produtos que necessitam de solubilidade em água. Obteve considerável teor de compostos fenólicos. A higroscopicidade apresentou valores considerados elevados podendo ser devido a forte interação entre açucares e moléculas de água relacionada aos terminais polares presentes nessas moléculas (Jaya e Das, 2004).

Tabela 2 - Resultados obtidos para solubilidade, teor de compostos fenólicos (CF), higroscopicidade e rendimento

\begin{tabular}{ccccccc}
\hline & $\mathbf{T}\left[{ }^{\circ} \mathbf{C}\right]$ & $\begin{array}{l}\mathbf{W} \\
{[\mathbf{L} / \mathbf{h}]}\end{array}$ & $\begin{array}{c}\text { Solubilidade } \\
(\boldsymbol{\%})\end{array}$ & $\begin{array}{c}\mathbf{C F} \\
(\mathbf{m g G A E} / \mathbf{g} \text { pó})\end{array}$ & $\begin{array}{c}\text { Higroscopicidade } \\
(\boldsymbol{\%})\end{array}$ & $\begin{array}{c}\text { Rendimento } \\
(\boldsymbol{\%})\end{array}$ \\
\hline $\mathbf{1}$ & 120 & 0,37 & 85,063 & 4,774 & 56,579 & 41,328 \\
\hline $\mathbf{2}$ & 158 & 0,36 & 100,000 & 5,318 & 56,512 & 42,425 \\
\hline $\mathbf{3}$ & 120 & 0,8 & 100,000 & 6,479 & 55,947 & 36,451 \\
\hline $\mathbf{4}$ & 158 & 0,8 & 94,577 & 6,227 & 56,107 & 43,119 \\
\hline $\mathbf{5}$ & 139 & 0,59 & 100,000 & 4,686 & 55,636 & 55,103 \\
\hline $\mathbf{6}$ & 139 & 0,59 & 90,0442 & 5,042 & 55,669 & 51,181 \\
\hline $\mathbf{7}$ & 139 & 0,57 & 99,110 & 4,576 & 55,635 & 51,908 \\
\hline
\end{tabular}

Os valores de compostos fenólicos do yacon atomizado foram próximos ao valor do yacon fresco, 10,42 mg GAE/g matéria seca (MS) (Pereira et al., 2016). Campos et al. (2012) ao estudarem 35 variedades de yacon encontraram valores entre 7,9 $\pm 0,8$ a 30,8 $\pm 0,1 \mathrm{mg}$ GAE/g MS. Em comparação com outros processos de conservação a secagem por atomização mostrouse eficiente para conservação dos compostos fenólicos do yacon, Oliveira (2010) ao branquear e decorticar no gelo o yacon, encontrou valores de $0,379 \pm 0,017 \mathrm{mg}$ de GAE/g de amostra e 0,751 $\pm 0,03 \mathrm{mg} / \mathrm{g}$, respectivamente.

A higroscopicidade apresentou valores menores que os da literatura para farinha de yacon, $94,37 \% \pm 0,03$, assim o processo de microencapsulação mostrou-se mais eficiente que a elaboração da farinha por reter menos água o que favorece seu armazenamento e comercialização (Medeiros, 2015).

A solubilidade apresentou valores compatíveis com a literatura, Brites et al. (2016) relatou valores acima de $90 \%$ para pós atomizados de yacon. Para guavira em pó utilizando goma arábica foi relatado uma variação de 74,62 \pm 5,97 a 83,16 \pm 0,77 \% (Chung, 2016).

Para o rendimento foi observado valores maiores que os descritos na literatura, (Nishi, 2012) ao microencapsular a polpa de yacon com maltodextrina e amido pré-gelatinizado encontrou valores de 23,20 a $39,03 \%$ e de 10,23 a $22,70 \%$ de rendimento, respectivamente.

Os resultados obtidos foram analisados estatisticamente. Os coeficientes de determinação, regressão e seus respectivos p-valores são apresentados na Tabela 3. Observa-se que as respostas apresentaram elevados coeficientes de determinação, indicando uma confiabilidade da análise estatística. 
Conforme Tabela 3, a vazão foi significativa em relação aos compostos fenólicos e higroscopicidade. Sendo que, aumentando a vazão acresceria a concentração de compostos fenólicos e diminuiria a higroscopicidade. A relação entre temperatura e vazão mostrou significância em relação à higroscopicidade demonstrando uma interação positiva.

Tabela 3 - Estimativa do p-valor e coeficiente de regressão (C.R.) para rendimento, teor de compostos fenólicos (CF), solubilidade e higroscopicidade

\begin{tabular}{|c|c|c|c|c|c|c|c|c|}
\hline & \multicolumn{2}{|c|}{$\begin{array}{c}\text { Rendimento } \\
{[\%]} \\
\left(\mathbf{R}^{2}=\mathbf{0 , 9 7}\right)\end{array}$} & \multicolumn{2}{|c|}{$\begin{array}{c}\text { CF [mg } \\
\text { GAE/ g pó] } \\
\left(\mathbf{R}^{2}=\mathbf{0 , 9 7 )}\right.\end{array}$} & \multicolumn{2}{|c|}{$\begin{array}{c}\text { Solubilidade } \\
{[\%]} \\
\left(\mathbf{R}^{2}=\mathbf{0 , 7 2}\right)\end{array}$} & \multicolumn{2}{|c|}{$\begin{array}{c}\text { Higroscopicidade } \\
{[\%]} \\
\left(\mathbf{R}^{2}=\mathbf{0 , 9 9}\right)\end{array}$} \\
\hline & C.R & p-valor & C.R. & $\begin{array}{c}\text { p- } \\
\text { valor }\end{array}$ & C.R. & $\begin{array}{l}\text { p- } \\
\text { valor }\end{array}$ & C.R. & $\mathrm{p}$-valor \\
\hline Média & 40,831 & 0,000 & 5,699 & 0,000 & 95,932 & 0,001 & 56,286 & 0,000 \\
\hline Curvatura & 11,899 & $0,017 *$ & 0,931 & 0,037 * & 1,104 & 0,837 & $-0,639$ & $0,000^{*}$ \\
\hline $\mathbf{T}(\mathbf{L})$ & 1,941 & 0,203 & 0,073 & 0,609 & 2,911 & 0,447 & 0,023 & 0,133 \\
\hline V (L) & $-1,045$ & 0,421 & 0,653 & $0,033^{*}$ & 1,845 & 0,613 & $-0,259$ & $0,001 *$ \\
\hline $\mathbf{T} \times \mathbf{V}$ & 1,392 & 0,313 & 0,199 & 0,243 & $-6,111$ & 0,188 & 0,056 & $0,026^{*}$ \\
\hline
\end{tabular}

Onde $\mathrm{T}$ é a temperatura em ${ }^{\circ} \mathrm{C}, \mathrm{V}$ é a vazão em $\mathrm{L} / \mathrm{h}, \mathrm{L}$ indica o termo linear. (*) indicam variáveis estatisticamente significativas, a $5 \%$ de significância.

Quando se observa a relação positiva entre compostos fenólicos e vazão pode-se inferir que sua interação se refere à encapsulação mais rápida, pois o extrato fica menos tempo exposto e sujeito à degradação, visto que, a sua pulverização estabiliza e preserva os compostos fenólicos de forma que sua concentração é maior em relação aos ensaios com vazões menores, sendo que compostos fenólicos são fotossensíveis. Como podemos observar a temperatura não foi significativa para as respostas no processo de secagem.

A partir dos resultados experimentais, analisou-se o ajuste das regressões por meio do teste $\mathrm{F}$, obtendo, estatisticamente, um bom ajuste da regressão $\left(\mathrm{F}_{\mathrm{cal}}>\mathrm{F}_{\text {tab }}-19,25\right)$ para higroscopicidade $\left(\mathrm{F}_{\mathrm{cal}}=678,06\right)$, gerando a superfícies de resposta (Figura 1). Não sendo possível o ajuste da regressão para solubilidade $\left(F_{\text {cal }}=1,29\right)$, rendimento $\left(F_{\text {cal }}=15,50\right)$ e compostos fenólicos $\left(\mathrm{F}_{\mathrm{cal}}=14,24\right)$.

Figura 1 - Superfície de resposta para higroscopicidade.

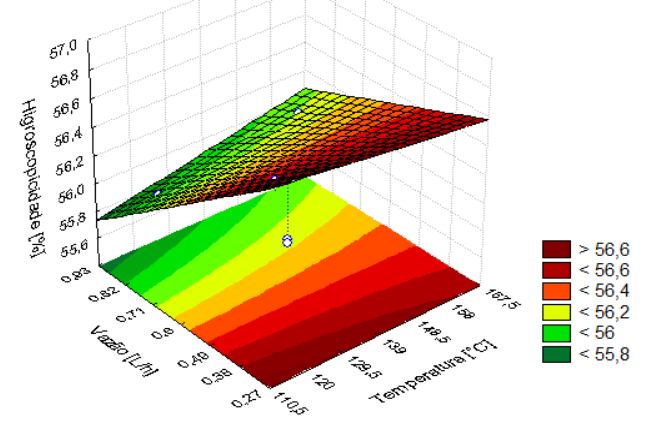


Ao analisar o gráfico de superfície de resposta conforme mostra a Figura 1 podemos confirmar o que foi demonstrado pelo coeficiente de regressão da Tabela 3 que, vazões mais elevadas, independente da temperatura, geram menores valores para a higroscopicidade. Em função da maior estabilidade do produto ao longo do tempo é desejável que este possua menor higroscopicidade, neste caso, a recomendação para a secagem da polpa de yacon em Spray Dryer seria o uso de uma vazão de alimentação acima de $0,6 \mathrm{~L} / \mathrm{h}$ em qualquer temperatura.

\section{CONSIDERAÇÕES FINAIS}

O processo de secagem por Spray Dryer do extrato de yacon se mostrou eficiente por apresentar um pó com elevada solubilidade, apreciável teor de compostos fenólicos e alto rendimento.

A vazão de alimentação influenciou na secagem, sendo que, maiores vazões levaram a menores valores para a higroscopicidade.

\section{REFERÊNCIAS BIBLIOGRÁFICAS}

BRITES, M. L.; MEIRA, S. M.; BRANDELLI, A.; NORENA, C. Z. Caracterização de pó do permeado do extrato de yacon obtido por ultrafiltração e desidratado por atomização. Ciênc. Agrotec., v. 40, n.5, p. 585-595, 2016.

CAMPOS, D.; BETALLELUZ-PALLARDEL, I.; CHIRINOS, R.; AGUILAR-GALVEZ, A.; NORATTO, G.; PEDRESCHI, R. Prebiotic effects of yacon (Smallanthus sonchifolius Poepp. \& Endl), a source of fructooligosaccharides and phenolic compounds with antioxidant activity. Food Chemistry., v. 135, p. 1592-1599, 2012.

CANO-CHAUCA, M.; STRINGHETA, P. C.; RAMOS, A. M.; CAL-VIDAL, J. Effect of the carriers on the microstructure of mango powder obtained by spray drying and its functional characterization. Innovative Food Science and Emerging Technologies, Oxford., v. 5, n. 4, p. 420-428, 2005.

CAStro, A.; CABAllero, M.; HERBAS, A.; CARBAllo, S. Antioxidants in yacon products and effect of long term storage. Food Science and Technology, Campinas., v. 32, n. 3, p. 432-435, 2012.

CHUNG, M. M. S. Polpa de guavira (Campomanesia cambessedeana Berg) desidratada em Spray Dryer: Efeitos das condições de processo e composição de alimentação nas propriedades físico químicas e atividade antioxidante. Tese (Mestrado em Engenharia e Ciências dos Materiais). Universidade de São Paulo, Pirassununga, 2016.

DELGADO, G. T. C.; TAMASHIRO W. M. S. C.; JUNIOR M. R. M.; PASTORE, G.M. Yacon (Smallanthus sonchifolius): a functional food. Plant foods for human nutrition., v. 68, n. 3, p. 222-228, 2013. 


\section{Congresso Brasileiro de Engenharia Química \\ em Iniciação Científica \\ UFSCar - São Carlos - SP \\ 16 a 19 de Julho de 2017}

JAYA, S.; DAS, H. Effect of maltodextrin, glycerol monostesrate and tricalcium phosphate on vacum dried mango powders properties. J. of Food Engineering., v. 63, p. 125-134, 2004.

MEDEIROS, M.F.D. Influência da composição química dos materiais no desempenho de secagem de polpas de frutas em leito de jorro. 233p. Tese (Doutorado em Engenharia Química), Universidade Estadual de Campinas, Campinas, 2001.

MEDEIROS, J. S. Elaboração e caracterização físico-química da farinha de batata yacon (Smallanthus sonchifolius). Trabalho de conclusão de curso (Curso Superior de Tecnologia em Alimentos), Instituto Federal de Educação, Ciência e Tecnologia do Rio Grande do Norte, Currais Novos, 2015.

NISHI, A. C. F. Extrato aquoso de yacon (Smallanthus sonchifolius) desidratado por atomização. 84p. Tese (Mestrado em Nutrição e Saúde), Universidade Federal de Goiás, Goiânia, 2012.

OLIVEIRA, L. A. Yacon (Smallanthus sonchifolius): compostos fenólicos totais e efeitos sobre a glicemia e estresse oxidativo em ratos diabéticos. Dissertação (Mestrado em Ciências Nutricionais) - Universidade Estadual Paulista "Júlio de Mesquita Filho", Programa de Pós-Graduação em Alimentos e Nutrição, Araraquara, 2010.

PEREIRA, J. A. R,; TEIXEIRA, M. C.; SACZK, A. A.; BARCELOS, M. F. P.; OLIVEIRA M. F.; ABREU, W. C. Total antioxidant activity of yacon tubers cultivated in Brazil. Ciência e Agrotecnologia., v. 40 n. 5, p. 596-605, 2016.

SCHEID, M.; PASTORE, G.; MARÓSTICA, M. Change in carbohydrate composition of fresh yacon (Smallanthus sonchifolius) roots during storage. A. of Nutrition \& Metabolism., v. 63, p.1640, 2013.

SINGLETON, V. L.; ORTHOFER, R.; LAMUELA-RAVENTÓS, R. M. Analysis of total phenols and other oxidation substrates and antioxidants by means of folin-ciocalteu reagent. Methods in enzymology., n. 299, p. 152-178, 1999.

STATISTICA. DATA ANALYSIS SOFTWARE SYSTEM, V.8.0, STAT-SOFT, INC., USA. 2008. Disponível em: < www.statsoft.com> Acesso em: 30 de agosto de 2016.

TAKENAKA, M.; YAN, X.J.; ONO, H.; YOSHIDA, M.; NAGATA, T.; NAKANISHI, T. Caffeic acid derivatives in the roots of yacon (Smallanthus sonchifolius). J. of Agricultural and Food Chemistry., v. 51, p. 793-796, 2003.

TONON, R. V.; BRABET, C.; PALLET, D.; BRAT, P.; HUBINGER M. D. Physicochemical and morphological characterisation of açai (Euterpe oleraceae Mart.) powder produced with different carrier agents. I. J. of food science \& technology., v. 44, n. 10, p. 1950-1958, 2009. 